\title{
The Test-Retest Reliability of Force Plate-Derived Parameters of the Countermovement Push-Up as a Power Assessment Tool
}

\author{
Gemma N. Parry, Lee C. Herrington, and lan G. Horsley
}

\begin{abstract}
Context: Muscular power output of the upper limb is a key aspect of athletic and sporting performance. Maximal power describes the ability to immediately produce power with maximal velocity at the point of release, impact, or takeoff, with research highlighting that the greater an athlete's ability to produce maximal power, the greater the improvement in athletic performance. Despite the importance of upper-limb power for athletic performance, there is presently no gold-standard test for upper-limb force development performance. Objective: The aim of this study was to investigate the test-retest reliability of force plate-derived measures of the countermovement push-up in active males. Design: Test-retest design. Setting: Controlled laboratory. Participants: Physically active college athletes (age 24 [3] y, height 1.79 [0.08] m, body mass 81.7 [9.9] kg). Intervention: Subjects performed 3 repetitions of maximal effort countermovement push-up trials on Kistler force plates on 2 separate test occasions 7 days apart. Main Outcome Measures: Peak force, mean force, flight time, rate of force development, and impulse were analyzed from the force-time curve. Results: No significant differences between the 2 trial occasions were observed for any of the derived performance measures. Intraclass correlation coefficient and within-subject coefficient of variation calculations indicated performance measures to have moderate to very high reliability (intraclass correlation coefficient $=.88-.98$ ), coefficient of variation $=5.5 \%-14.1 \%)$. Smallest detectable difference for peak force $(7.5 \%)$, mean force $(8.6 \%)$, and rate of force development (11.2\%) were small to moderate. Conclusion: Force platform-derived kinetic parameters of countermovement push-up are reliable measurements of power in college-level athletes.
\end{abstract}

Keywords: upper-limb power, meaningful difference, force-time curve

Upper-limb muscular power output is a fundamental and highly desirable characteristic across a number of explosive short-duration sports. ${ }^{1,2}$ Because upper-limb muscle power output is considered to be a principle movement skill and a key aspect of sporting performance and athletic ability, it is important to identify ways of assessing an athlete's ability to produce upper-limb force in order to not only understand an individual's performance potential but also monitor training program effects, long-term development, and talent identification.

Despite the importance of upper-limb muscular power, there is presently no gold-standard test of upper-limb force development performance. Previously, medicine ball throws, bench press, and timed push-ups have been utilized to monitor and assess training regime effectiveness with regard to upper-limb muscular performance. ${ }^{3-7}$ There is a paucity of research analyzing upper-limb power production via a plyometric push-up, with only a few studies investigating force plate-derived kinetic data to assess upper-limb power output. ${ }^{5-7}$ There is evidence of moderate to high reliability with strong test-retest correlations (intraclass correlation coefficient [ICC] = .84-.96) for the kinetic parameters of force, impulse, and rate of force development (RFD) between testing sessions for bench press and explosive push-up in active male individuals ${ }^{6}$; however, due to a focus on heavy resistance exercise effects, the

Parry is with GB Boxing, English Institute of Sport, Sheffield, United Kingdom; and the Human Performance Laboratory, Directorate of Sport, Exercise and Physiotherapy, School of Health Sport and Rehabilitation Sciences, University of Salford, Salford, United Kingdom. Herrington is with the School of Health, Sport and Rehabilitation Sciences, University of Salford, Manchester, United Kingdom. Herrington and Horsley are with Physiotherapy Department, English Institute of Sport, Manchester, United Kingdom. Parry (g.parry1@edu.salford.ac.uk) is corresponding author. stretch-shortening cycle movement, and no reported outcome on vertical displacement, it is unknown whether these parameters can be reliably measured during countermovement push-up (CMPU) performance. Researchers ${ }^{7}$ have also reported a trend of high reliability (ICC $=.85-.97)$ for peak force (PF) during 4 plyometric push-up variations. Furthermore, a recent study solely investigating the reliability of a plyometric push-up ${ }^{5}$ reported excellent between-test reliability (ICC $=.8-.96$ ) for PF for subelite rugby league players. However, RFD did not demonstrate the same reliability (ICC $\leq .84)$. Despite the availability of commensurable literature, Hogarth et al ${ }^{5}$ and Koch et al ${ }^{7}$ attributed result disparities to lack of subject familiarization with the technique.

Timed push-ups have been highlighted as poor for assessment of force-time performance due to an athlete only being required to move $60 \%$ of their body mass-as opposed to the $100 \%$ required for power ${ }^{8}$ - and are arguably more likely to be a measure of muscular endurance. Cronin et $\mathrm{al}^{9}$ demonstrated that a countermovement bench press throw increased peak throw power by $11.7 \%$ across a range of $30 \%$ to $80 \%$ of an individual's repetition maximum, indicating that the stretch-shortening cycle in the upper limb may have been enhanced in relation to power output. Although the feet act as a pivot point by remaining in contact with the floor, the explosive similarities and similar displacement of body mass through a vertical plane in both the countermovement jump (CMJ) and CMPU make it reasonable to assume that the force-time characteristics witnessed and reported in the CMJ literature may also apply to the CMPU as an evaluator of performance.

Generation of upper-limb power is an important factor across a range of sports. ${ }^{1,2}$ Valid and reliable protocols for its assessment and development are valuable measurements that can be utilized to impact sporting performance. While a performance test might demonstrate high reliability, this does not always directly represent 
a substantial change in performance due to random error and learning effect measurement error. ${ }^{10}$ Smallest detectable differences (SDDs) allow practitioners to deduce meaningful changes in performance rather than changes due to measurement errors. ${ }^{11}$ Therefore, there is a need to further understand and rationalize the use of CMPU as a tool to measure upper-limb power, which could potentially give coaches and practitioners a reliable measurement tool that provides recognized meaningful differences in upper-limb power output within sporting and active populations.

Further research is required to understand relationships between force-time characteristics because at present there is a lack of published information that reports the reliability of CMPU as a testing protocol for upper-limb performance. The aim of this study, therefore, was to investigate the test-retest reliability of force plate-derived measures of CMPU in active males. A secondary aim was to explore the SDD that occurred between trials for meaningful changes.

\section{Methods}

\section{Experimental Approach to the Problem}

A single-group, repeated-measure design was used, with 3 repetitions of maximal-effort CMPU on 2 separate testing sessions 7 days apart.

\section{Participants}

A total of 10 male college athletes (age 24 [3] y; height 1.79 [0.08] m; body mass 81.7 [9.9] kg) with a minimum of 1 year of Olympic lifting training history participated in this study. The institutional review board approved the investigation and preceding testing, and all subjects provided written informed consent.

\section{Procedures}

Three repetitions of maximal-effort CMPU trials, with 2 minutes of rest between trials, were completed on 2 separate occasions at the same time of day, 7 days apart, in order to determine reliability when following a standardized protocol. Trials were performed on 2 portable force plates (Kistler type 9286; Kistler, Winterthur, Switzerland), and Bioware Software (version 5.11; Bioware Software, Switzerland) was used to collect kinetic data at a sampling frequency of $1000 \mathrm{~Hz}$. During testing, the participant's weight was evenly distributed with a force plate beneath each hand, the shoulders at 90 degrees of flexion, with the torso, legs and elbows extended and malleoli and feet together. Following a 3-second countdown, the participant immediately lowered his torso rapidly toward the force plate, then immediately pressed vertically as high as possible, aiming for maximal height and trunk elevation, with elbows extended and hands clearing the plate, and landing back on the force plate with both hands at the same time (Figure 1). Performance variables (PF [in newtons]; mean force, MF [in newtons]; peak RFD [in newtons per second], flight time [in seconds], and impulse [in newton per second]) were taken from the force-time curve (Figure 2), and data were filtered using a Butterworth low-pass filter at $8 \mathrm{~Hz}$. The greatest $\mathrm{PF}$ determined the best CMPU effort for each participant.

\section{Statistical Analysis}

Statistical analysis was carried out using SPSS version 25 (Chicago, IL) for Windows. Paired sample $t$ tests were performed

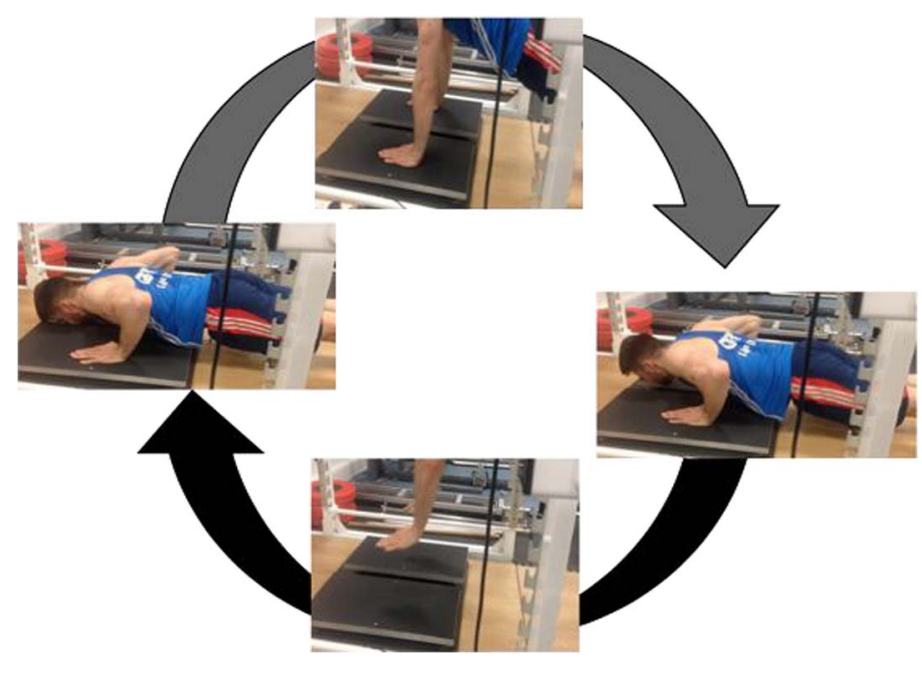

Figure 1 - Example of countermovement push-up.

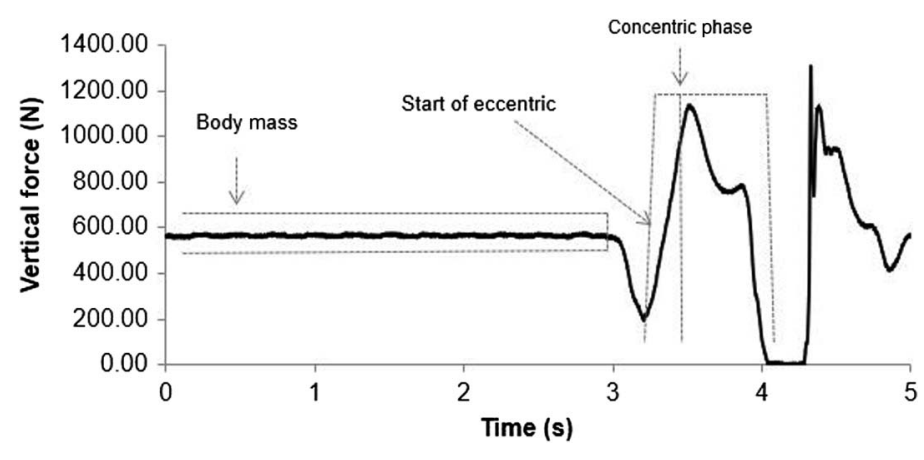

Figure 2 - Force trace from a subject performing countermovement push-up.

to deduce any significant changes in performance measures between trial sessions. ICC and within-subject coefficient of variation $(\mathrm{CV} \%)$ were calculated with $95 \%$ confidence intervals to determine relationships between test-retest reliability and reproducibility. SEM and SDD were also included to represent and identify the smallest clinically worthwhile change that was statistically significant using SD [pooled] $\times \sqrt{ } 1-$ ICC for SEM and $1.96 \times \sqrt{ } 2 \times$ SEM for SDD. ${ }^{12}$ Data are presented as mean (SD), and significance is set at $P \leq .05$.

\section{Results}

Descriptive and reliability statistics are presented for each derived parameter in Table 1. Paired sample $t$ tests indicated no significant differences between the 2 trials $(P>.05)$ for any of the derived performance measures. ICC and within-subject coefficient of variation calculations indicated that the performance measures had moderate to very high reliability (ICC $=.88-.98, \mathrm{CV} \%=5.5-$ 14.1). Within-subject calculations appeared to demonstrate moderate to high reliability $(\mathrm{CV}=1.7 \%-6.9 \%$, ICC $=.96-.98)$ for the derived parameters of $\mathrm{PF}$, flight time, MF, and impulse; only RFD was outside acceptable standards of reliability $(\mathrm{CV}=14.1 \%$, $\mathrm{ICC}=.87)$. SDD for PF $(7.5 \%), \mathrm{MF}(8.6 \%)$, and RFD $(11.2 \%)$ was small to moderate. 
Table 1 Values of Reliability Statistics for Countermovement Push-Up-Derived Parameters $(n=10)$

\begin{tabular}{|c|c|c|c|c|c|c|c|}
\hline Derived parameter & Trial 1 & Trial 2 & SEM & CV\% & ICC & SDD & SDD\% \\
\hline Flight time, $\mathrm{s}$ & $0.3(0.0)$ & $0.3(0.0)$ & 0.0 & 6.9 & .964 & 0.0 & 0.0 \\
\hline Peak force, $\mathrm{N}$ & $1171(41)$ & $1140(48)$ & 31.1 & 3.9 & .963 & 86.3 & 7.5 \\
\hline Mean force, $\mathrm{N}$ & $589(14)$ & $587(6.4)$ & 1.7 & 1.7 & .967 & 50.3 & 8.6 \\
\hline Rate force development, N/s & $2585(137)$ & $2599(147)$ & 105.1 & 14.1 & .878 & 291.3 & 11.2 \\
\hline Impulse, $\mathrm{N} \cdot \mathrm{s}$ & $77.5(10.9)$ & $73(7.5)$ & 7.1 & 5.5 & .983 & 19.7 & 26.1 \\
\hline
\end{tabular}

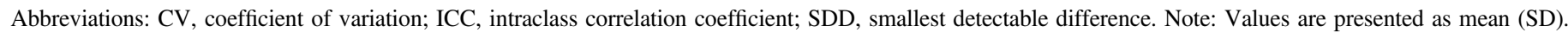

\section{Discussion}

The focus of this article was the test-retest reliability of the force time-derived parameters of a CMPU in college-level athletes. Presently, there are no clear, acceptable standards of reliability for a CMPU. However, CV\% $<10 \%$ and ICC values of $>75 \%$ have been shown to be demonstrative of good reliability for measures obtained during CMJs. ${ }^{2}$ The findings of this study indicate that CMPU force plate-derived parameters are reliable indicators of performance in college-level athletes. Previous research $^{5-7}$ has reported comparable values of moderate to high test-retest reliability for force time-derived parameters from variations of push-ups within Rugby League $(\mathrm{CV}=2.3 \%-11 \%)$ and mixed cohorts of recreationally active participants and active duty marines ( $\mathrm{ICC}=.85 \%-.97 \%)$, with $\mathrm{MF}(\mathrm{CV}=4.8 \%)$ demonstrating the best reliability. Similar to the mixed-cohort participants, ${ }^{6,7}$ the participants in this investigation were college athletes with a minimum 1-year history of Olympic lifting and some plyometric training, and although they may not have participated in pushup exercises as regularly as elite athletes, a high reliability (ICC $=.87 \%-.98 \%$ ) of the derived parameters between test sessions was still demonstrated. This is an important finding because it suggests that CMPU force plate-derived kinetic data are reliable performance measure in elite, trained, and recreationally trained college populations.

Given the widely reported body of evidence for the high reliability of peak measures of force and power within the CMJ literature, it is not surprising that a parallel high reliability for the parameters of $\mathrm{MF}(\mathrm{CV}=1.7 \%)$ and $\mathrm{PF}(\mathrm{CV}=3.9 \%)$ was found for CMPU. The higher reliability of the average value is potentially due to the ultimate PF values being unaffected by negligible variations in PF. ${ }^{10}$ Despite the reported reliability of CMPU parameters, RFD demonstrated poor reliability within this study $(\mathrm{CV}=14.9 \%)$. In addition, although the results for ICC (ICC $=.87 \%$ ) were comparable with another study, ${ }^{6}$ it is questionable whether it is a reliable parameter. Likewise, RFD demonstrated poor reliability within the previously mentioned studies. ${ }^{5-7}$ Although there is a paucity of additional commensurable literature for CMPU, our findings are consistent with poor RFD reliability values $(\mathrm{CV}=11.8 \%$, ICC $=.75 \%)$ presented within the $\mathrm{CMJ}$ literature $^{2}$; given the importance of RFD to fast, forceful muscle contraction, ${ }^{10}$ the results should be interpreted with caution until RFD reliability is clearly established. Methods for measuring CMJ performance affect RFD reliability; impulse methods have been shown to have lower associated errors to deduce RFD comparative with flight-time methods, ${ }^{2,10}$ which may account for the poor reported reliability of RFD. When assessing force, practitioners should be looking for changes from $8 \%$ to $9 \%$ in order to determine meaningful change, particularly if they are using CMPU to monitor training program effects and long-term athlete development because tests that are deemed reliable are not necessarily the most effective for monitoring performance changes.

\section{References}

1. Sander A, Keiner M, Wirth K, Schmidtbleicher D. Influence of a 2-year strength training programme on power performance in elite youth soccer players. Eur J Sport Sci. 2013;13(5):445-451. PubMed ID: 24050460 doi:10.1080/17461391.2012.742572

2. Cormack SJ, Newton RU, McGuigan MR, Doyle TLA. Reliability of measures obtained during single and repeated countermovement jumps. Int J Sports Physiol Perform. 2008;3(2):131-144. PubMed ID: 19208922 doi:10.1123/ijspp.3.2.131

3. Stockbrugger BA, Haennel RG. Validity and reliability of a medicine ball explosive power test. J strength Cond Res. 2001;15(4):431-438. PubMed ID: 11726253

4. McGuigan MR, Wright GA, Fleck SJ. Strength training for athletes: does it really help sports performance? Int J Sports Physiol Perform. 2012;7(1):2-5. PubMed ID: 22461461 doi:10.1123/ijspp.7.1.2

5. Hogarth L, Deakin G, Sinclair W. Are plyometric push-ups a reliable power assessment tool? J Aust Strength Cond. 2013;21:67-69.

6. Hrysomallis C, Kidgell D. Effect of heavy dynamic resistive exercise on acute upper-body power. J Strength Cond Res. 2001;15(4): 426-430. PubMed ID: 11726252

7. Koch J, Riemann BL, Davies GJ. Ground reaction force patterns in plyometric push-ups. J Strength Cond Res. 2012;26(8):2220-2227. PubMed ID: 21986698 doi:10.1519/JSC.0b013e318239f867

8. Ferreira LC, Schilling BK, Weiss LW, Fry AC, Chiu LZF. Reach height and jump displacement: implications for standardization of reach determination. J Strength Cond Res. 2010;24(6):1596-1601. PubMed ID: 20508464 doi:10.1519/JSC.0b013e3181d54a25

9. Cronin J, McNair PJ, Marshall RN. Developing explosive power: a comparison of technique and training. J Sci Med Sport. 2001;4(1):5970. PubMed ID: 11339494 doi:10.1016/S1440-2440(01)80008-6

10. Nibali ML, Tombleson T, Brady PH, Wagner P. Influence of familiarization and competitive level on the reliability of countermovement vertical jump kinetic and kinematic variables. J Strength Cond Res. 2015;29(10):2827-2835. PubMed ID: 26398700 doi:10.1519/JSC. 0000000000000964

11. Comfort P, McMahon JJ. Reliability of maximal back squat and power clean performances in inexperienced athletes. J Strength Cond Res. 2015;29(11):3089-3096. PubMed ID: 25559912 doi:10.1519/ JSC.0000000000000815

12. Munro A, Herrington L, Carolan M. Reliability of 2-dimensional video assessment of frontal-plane dynamic knee valgus during common athletic screening tasks. J Sport Rehabil. 2012;21(1):7-11. PubMed ID: 22104115 doi:10.1123/jsr.21.1.7 